\title{
Continuous Monotonic Decomposition of Some Standard Graphs by using an Algorithm
}

\author{
C. Sujatha, A. Manickam
}

\begin{abstract}
In this paper we elaborate an algorithm to compute the necessary and sufficient conditions for the continuous monotonic star decomposition of the bipartite graph $K_{m, r}$ and the number of vertices and the number of disjoint sets. Also an algorithm to find the tensor product of $P_{n} \times P_{s}$ has continuous monotonic path decomposition. Finally we conclude that in this paper the results described above are complete bipartite graphs that accept Continuous monotonic star decomposition. There are many other classes of complete tripartite graphs that accept Continuous monotonic star decomposition. In this research article Extended to complete m-partite graphs for grater values of m. Also the algorithm can be developed for the tensor product of different classes such as $C_{n}, W_{n}, K_{1, n}$ with $P_{n}$

Keywords: Complete bipartite graph, Continuous monotonic star decomposition, Tensor product. Mathematical subject classification: 03B52, 03E72, 08A72.
\end{abstract}

\section{INTRODUCTION}

A simple graph with the property that there is a path between every pair of vertices is known as a connected graph. The degree of a vertex $v$ of any graph is the number of edges incident with $v$ and is denoted by $d(v)$ and the distance between the two vertices $u$ and $v$ of $G$ is the length of the shortest $v-u$ path in $G$ and is denoted by $d(u, v) . \quad\left\{G_{i} / i=1,2, \ldots n\right\} \quad$ be $\quad$ a collection of edge-disjoint sub graphs of $G$ such that $E(G)=E\left(G_{1}\right) \cup E\left(G_{2}\right) \cup \ldots \cup E\left(G_{n}\right)$, then the collection $\left\{G_{i}\right\}$ is called a decomposition of $G$. If each $G_{i}$ is connected and $\left|E\left(G_{i}\right)\right|=i$ for each $i=1,2, \ldots, n$, then it is called a continuous monotonic decomposition of $G$. A complete graph with vertices $n \varepsilon N$, denoted by $K_{n}$ is a connected simple graph with every vertex is connected with every other vertex by an edge [1]. A graph with $n$ vertices

Revised Manuscript Received on July 13, 2019.

* Correspondence Author

C. Sujatha, Principal, Professor of Mathematics, MaruduPandiyar College, Vallam Post, Thanjavur (Tamil Nadu), India. E-mail: sujatha2276@gmail.com

A. Manickam, Associate Professor of Mathematics, Vel Tech Rangarajan Dr. Sagunthala R\&D Institute of Science and Technology, (Deemed to be University), Avadi-Veltech Chennai (Tamil Nadu), India. E-mail: dramanickam@veltech.edu.in, manickammaths2011@gmail.com

(c) The Authors. Published by Blue Eyes Intelligence Engineering and Sciences Publication (BEIESP). This is an open access article under the CC BY-NC-ND license (http://creativecommons.org/licenses/by-nc-nd/4.0/)

$$
\begin{aligned}
& v_{1}, v_{2}, \ldots v_{n}, \quad \text { where } n>=3, \quad \text { and edges } \\
& \left\{v_{1}, v_{2}\right\}\left\{v_{1}, v_{2}\right\},\left\{v_{2}, v_{3}\right\}, \ldots,\left\{v_{n-1}, v_{n}\right\},\left\{v_{n}, v_{1}\right\} \text { is }
\end{aligned}
$$

known as a cycle, $C_{n}$.
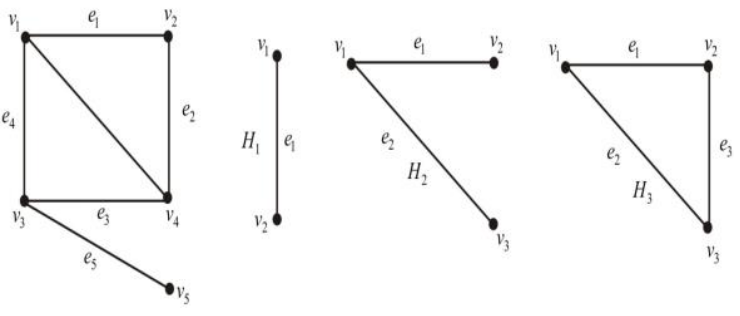

Fig.1.

\section{STAR DECOMPOSITION OF A GRAPH}

Let $G=(V, E)$ be a connected simple graph of order $m$ and size $n$. If A decomposition $\left.G_{1}, G_{2}, \ldots, G_{n}\right)$ of $G$ is said to be a continuous monotonic decomposition (CMD) if each $G_{i}$ is connected and $\left|E\left(G_{i}\right)\right|=i \forall i \varepsilon N$.[2].Introduced Ascending Sub graph Decomposition(ASD) as a decomposition of $G$ into subgraphs $G_{i}$ (not necessarily connected) and is isomorphic to a proper sub graph $G_{i+1}$. [3] Introduced a new concept known as continuous monotonic decomposition of Graphs [3]. If $G$ admits a CMD, $\left\{G_{1}, G_{2}, \ldots, G_{n}\right\} \mid \forall n \varepsilon N$, where each $G_{i}$ is a star, then we say that $G$ admits Continuous Monotonic Cycle Decomposition (CMCD)[4].

\section{CONTINUOUS MONOTONIC DECOMPOSITION OF COMPLETE BIPARTITE GRAPHS $\mathrm{K}_{\mathrm{m}, \mathrm{r}}$}

A graph $G$ is a bipartite graph if $V(G)$ can be partitioned into two subsets $U$ and $V$, called bipartite sets such that every edge of $G$ joins a vertex of $u$ and a vertex of $v$. In a bipartite graph, if every vertex of $u$ is adjacent to every vertex of $v$, then such graph is called complete bipartite graph. A complete bipartite graph with $|U|=s$ and $|V|=t$ is denoted by $K_{s, t}$. If either $S=1$ or $t=1$ then $K_{s, t}$ is a star. Continuous Monotonic Decomposition of a wide variety of graphs had been studied by $[4,5]$.

Published By:

Blue Eyes Intelligence Engineering \& Sciences Publication

(C) Copyright: All rights reserved.

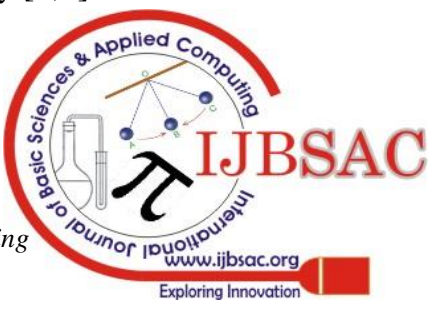




\section{Continuous Monotonic Decomposition of Some Standard Graphs by using an Algorithm}

\section{TENSOR PRODUCT}

For two graphs $G$ and $H$, the tensor product $G \times H$ has vertex set $V(G) \times V(H)$ in which $\left(l_{1}, m_{1}\right)$ and $\left(l_{2}, m_{2}\right)$ are adjacent whenever $l_{1} l_{2} \varepsilon E(G)$ and $m_{1} m_{2} \varepsilon E(H)$.

\subsection{Theorem}

Let $G$ be a connected simple graph of order $r$ and size $s$. Then $G$ admits a CMD $H_{1}, H_{2}, \ldots, H_{n}$ if and only if $q={ }^{(n+1)} C_{2}$.

\subsection{Theorem}

$K_{n}^{+}$admits a Continuous monotonic star decompositionfor all $n \geq 1$.

\subsection{Theorem}

(i) $K_{n, 2 n+1}$ admits Continuous monotonic star decomposition for $n \geq 1$

(ii) $K_{n+1,2 n+1}$ admits Continuous monotonic star decomposition for $n \geq 1$

\subsection{Theorem}

There is an edge decomposition of $G$ such that each partition class is a star in $G$ if and only if $G$ is bipartite.

4.5. Lemma

Let $G$ be a edge disjoint union of stars $S_{i+1}, S_{i+2}, \ldots, S_{i+k}$ for some $k>0$ such that $q=i(i+1) / 2$. Then can be decomposed into stars $S_{1}, S_{2}, \ldots, S_{i}$.

\subsection{Theorem}

The complete bipartite graph $K_{m, r}(m \leq r)$ can be decomposed into stars $S_{1}, S_{2}, \ldots, S_{2 n}$ (CMSD) if and only if $m=n-1$ and $r=2 n+1+j$ where $i, j>0$ such that $n=i(j+1) /(j-2 i)$.

\subsubsection{Algorithm}

1. $\left(K_{m, r}\right)$

Step 1: Initially enter the values $m, r, x$

Step 2: Read the value of $x$

Step 3: for $i=1$ to $n$ do

Step 4: for $j=1$ to $n$ do

(i) if $(j>2 * i)$ then

(ii) compute $y=i(j+1) /(j-2 * i)$

(iii) compute $m=y-i$

(iv) compute $r=2 * y+1+j$

Step 5: if $(m<=r \& \& m>0)$

Step 6: Print $i, j, y, m$ and $r$

Step 7: Go to step -3 until $i>n$

Step 8: Stop;
Step 9: End

Output

Enter the value of $(n=15)$

Table 1

\begin{tabular}{|l|l|l|l|l|}
\hline $\boldsymbol{I}$ & $\boldsymbol{J}$ & $\mathbf{2 n}$ & $\boldsymbol{M}$ & $\boldsymbol{R}$ \\
\hline 1 & 3 & 8 & 3 & 12 \\
\hline 1 & 5 & 4 & 1 & 10 \\
\hline 2 & 5 & 24 & 10 & 30 \\
\hline 2 & 6 & 14 & 5 & 21 \\
\hline 2 & 9 & 8 & 2 & 18 \\
\hline 2 & 14 & 6 & 1 & 21 \\
\hline 3 & 7 & 48 & 21 & 56 \\
\hline 3 & 9 & 20 & 7 & 30 \\
\hline 3 & 13 & 12 & 3 & 26 \\
\hline 4 & 9 & 80 & 36 & 90 \\
\hline 4 & 10 & 44 & 18 & 55 \\
\hline 4 & 11 & 32 & 12 & 44 \\
\hline 4 & 12 & 26 & 9 & 39 \\
\hline 4 & 14 & 20 & 6 & 35 \\
\hline 5 & 11 & 120 & 55 & 132 \\
\hline 6 & 13 & 168 & 78 & 182 \\
\hline 6 & 14 & 90 & 39 & 105 \\
\hline
\end{tabular}

4.7. Continuous monotonic decomposition of Tensor Product of $P_{n} \times P_{s}$

\subsubsection{Theorem}

For any integer $n, P_{n} \times K_{2}$ has a CMD $\left\{H_{1}, H_{2}, \ldots, H_{m}\right\}$ if and only if there exist an integer $m$ satisfying the following properties.

(i). $m=4 k$ or $m=4 k-1(k \geq 1, k \varepsilon Z)$

(ii) $m(m+1) / 2=4 n-4$

\subsubsection{Lemma}

(i) Let $m \equiv 0(\bmod 4)$. Two copies of the set $\{1,2, \ldots, m\}$ can be partitioned into four sets $S_{1}, S_{2}, S_{3}$ and $S_{4}$ such that $\sum_{a \varepsilon S_{1}} a=\sum_{b \varepsilon S_{1}} b=\sum_{c \varepsilon S_{1}} c=\sum_{d \varepsilon S_{1}} d=n-1$. Here $m(m+1)=4 n-4$.

(ii)Let $m+1 \equiv 0$ (mod 4$)$. Two copies of the set $\{1,2, \ldots, m\}$ can be partitioned into four sets $S_{1}, S_{2}, S_{3}$ and $S_{4}$ such that $\sum_{a \varepsilon S_{1}} a=\sum_{b \varepsilon S_{1}} b=\sum_{c \varepsilon S_{1}} c=\sum_{d \varepsilon S_{1}} d=n-1$. Here $m(m+1)=4 n-4$.

\subsubsection{Theorem}

For any integer $n, P_{n} \times K_{2}$ has two copies of continuous monotonic decomposition $\left\{H_{1}, H_{2}, \ldots, H_{m}\right\}$ if and only if there exist an integer $m$ satisfying the following properties.

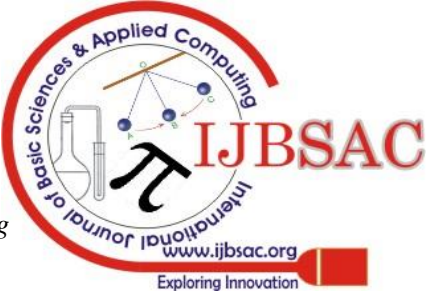




$$
\begin{aligned}
& \text { (i) } m=4 k \text { or } m=4 k-1(k \geq 1, k \varepsilon Z) \\
& \text { (ii) } m(m+1)=4 n-4
\end{aligned}
$$

\section{Proof}

Let $G=P_{n} \times P_{3}$. By definition $|E(G)|=4 n=4$. Assume $P_{n} \times P_{3}$ has two copies of CMD $\left\{H_{1}, H_{2}, \ldots . ., H_{m}\right\}$. We have $|E(G)|=2 x^{(n+1)} C_{2}$. Here $4 n-4=2 x^{(n+1)} C_{2}$. Since $P_{n} \times P_{3}$ has two copies of CMD, $4 n-4=2 \times(1+2+\ldots \ldots+m)=2 \times m(m+1) / 2=m(m+1)$.

Hence $m(m+1)=4 k(K \geq 1, k \varepsilon Z)$. Now either $m=4 k$ or $m=4 k-1$.

Conversely assume $m(m+1)=0(\bmod 4)$. Let $G=P_{n} \times P_{3} . \quad$ Let $\quad P_{n}=\left(u_{1}, u_{2}, \ldots \ldots, u_{n}\right)$, $P_{3}=\left(v_{1}, v_{2}, v_{3}\right)$.

Define $w_{i j}=\left(u_{i}, v_{j}\right)$, where $1 \leq i \leq n, 1 \leq j \leq 3$.

\section{Case 1: Suppose $n$ is odd.}

Define

$T_{1}=\left\{w_{i 1}, w_{(i+1) 2}: 1 \leq i \leq n-1, i-\right.$ odd $\}$

$\cup\left\{w_{i 2}, w_{(i+1) 1}: 1 \leq i \leq n, i-e v e n\right\}$.

$T_{2}=\left\{w_{i 2}, w_{(i+1) 1}: 1 \leq i \leq n-1, i-\right.$ odd $\}$

$\cup\left\{w_{i 1}, w_{(i+1) 2}: 1 \leq i \leq n, i-e v e n\right\}$.

$T_{3}=\left\{w_{i 2}, w_{(i+1) 3}: 1 \leq i \leq n-1, i-\right.$ odd $\}$

$\cup\left\{w_{i 3}, w_{(i+1) 2}: 1 \leq i \leq n, i-e v e n\right\}$.

$T_{4}=\left\{w_{i 3}, w_{(i+1) 2}: 1 \leq i \leq n-1, i-\right.$ odd $\}$

$\cup\left\{w_{i 2}, w_{(i+1) 3}: 1 \leq i \leq n, i-e v e n\right\}$.

Here $\quad\left|T_{1}\right|=\left|T_{2}\right|=\left|T_{3}\right|=\left|T_{4}\right|=n-1$.

Also

$\left|T_{1}\right|+\left|T_{2}\right|={ }^{(m+1)} C_{2}$ and $\left|T_{3}\right|+\left|T_{4}\right|={ }^{(m+1)} C_{2}$.

By Lemma $10, \quad\{1,2, \ldots, m\}=S_{1} \cup S_{2} \quad$ and $\{1,2, \ldots, m\}=S_{3} \cup S_{4}$

$\Sigma_{a \varepsilon S_{1}} a=\Sigma_{b \varepsilon S_{1}} b=\Sigma_{c \varepsilon S_{1}} c=\Sigma_{d \varepsilon S_{1}} d$. Decompose $T_{1}, T_{2}$ into trees $\left\{H_{i}\right\}$ as follows: $T_{1}=\bigcup_{i \varepsilon S_{1}} H_{i}$ and $T_{2}=\bigcup_{i \varepsilon S_{2}} H_{i} \cdot\left|E\left(H_{i}\right)\right|=i$, where $1 \leq i \leq m$. Also decompose $T_{3}, T_{4}$ into trees $\left\{H_{i}\right\}$ as follows: $T_{3}=\bigcup_{i \varepsilon S_{3}} H_{i} \quad$ and $\quad T_{4}=\bigcup_{i \varepsilon S_{4}} H_{i}$

$\left|E\left(H_{i}\right)\right|=i$, where $1 \leq i \leq m$. Clearly two copies of $\left\{H_{1}, H_{2}, \ldots . H_{m}\right\}$ forms the continuous monotonic decompositions of $P_{n} \times P_{3}$.

\section{Case 2: Suppose $\boldsymbol{n}$ is even.}

Define $T_{1}, T_{2}, T_{3}$ and $T_{4}$. As the case 1 , two copies of $\left\{H_{1}, H_{2}, \ldots . ., H_{m}\right\}$ forms the continuous monotonic decompositions of $P_{n} \times P_{3}$.

\subsubsection{Algorithm}

Step 1: Initially enter the values $n, m, a, b$

Step 2: Read the values of $m, n$

Step 3: While $(a \leq m-1)$

Step 4: Store $a=1 ; b=2$;

(i) $\quad$ For $i=1$ to $n$ do

(ii) Line $(i, a)-(i+1, b)$

(iii) Swap $(a, b)$

Step 5: Store $a=2 ; b=1$

(i) $\quad$ For $i=1$ to $n$ do

(ii) Line $(i, a)-(i+1, b)$

(iii) Swap $(a, b)$

Step 6: Goto Step 3 Until $(a \leq m-1)$

Step 7: Stop

Step 8: End

Output.

\begin{tabular}{|c|c|c|c|c|c|c|c|}
\hline \multicolumn{2}{|c|}{$T_{1}$} & \multicolumn{2}{|c|}{$T_{2}$} & \multicolumn{2}{|c|}{$T_{3}$} & \multicolumn{2}{|c|}{$T_{4}$} \\
\hline From & To & From & To & From & To & From & To \\
\hline$(1,1)$ & $\begin{array}{l}(2, \\
2)\end{array}$ & $(1,2)$ & $\begin{array}{l}(2, \\
1)\end{array}$ & $(1,2)$ & $\begin{array}{l}\text { (2, } \\
3)\end{array}$ & $(1,3)$ & $\begin{array}{l}(2, \\
2)\end{array}$ \\
\hline$(2,2)$ & $\begin{array}{l}\text { (3, } \\
1)\end{array}$ & $(2,1)$ & $\begin{array}{l}\text { (3, } \\
2)\end{array}$ & $(2,3)$ & $\begin{array}{l}\text { (3, } \\
2)\end{array}$ & $(2,2)$ & $\begin{array}{l}\text { (3, } \\
3)\end{array}$ \\
\hline$(3,1)$ & $\begin{array}{l}(4, \\
2)\end{array}$ & $(3,2)$ & $\begin{array}{l}\text { (4, } \\
1)\end{array}$ & $(3,2)$ & $\begin{array}{l}\text { (4, } \\
3)\end{array}$ & $(3,3)$ & $\begin{array}{l}4, \\
2)\end{array}$ \\
\hline$(4,2)$ & $\begin{array}{l}\text { (5, } \\
1)\end{array}$ & $(4,1)$ & $\begin{array}{l}(5, \\
2)\end{array}$ & $(4,3)$ & $\begin{array}{l}(5, \\
2)\end{array}$ & $(4,2)$ & $\begin{array}{l}\text { (5, } \\
3)\end{array}$ \\
\hline$(5,1)$ & $\begin{array}{l}\text { (6, } \\
2)\end{array}$ & $(5,2)$ & $\begin{array}{l}\text { (6, } \\
1)\end{array}$ & $(5,2)$ & $\begin{array}{l}\text { (6, } \\
3)\end{array}$ & $(5,3)$ & $\begin{array}{l}\text { (6, } \\
2)\end{array}$ \\
\hline$(6,2)$ & $\begin{array}{l}\text { (7, } \\
1)\end{array}$ & $(6,1)$ & $\begin{array}{l}\text { (7, } \\
2)\end{array}$ & $(6,3)$ & $\begin{array}{l}\text { (7, } \\
2)\end{array}$ & $(6,2)$ & $\begin{array}{l}\text { (7, } \\
\text { 3) }\end{array}$ \\
\hline$(7,1)$ & $\begin{array}{l}(8, \\
2)\end{array}$ & $(7,2)$ & $\begin{array}{l}\text { (8, } \\
1)\end{array}$ & $(7,2)$ & $\begin{array}{l}\text { (8, } \\
3)\end{array}$ & $(7,3)$ & $\begin{array}{l}(8, \\
2)\end{array}$ \\
\hline$(8,2)$ & $\begin{array}{l}\text { (9, } \\
1)\end{array}$ & $(8,1)$ & $\begin{array}{l}(9, \\
2)\end{array}$ & $(8,3)$ & $\begin{array}{l}(9, \\
2)\end{array}$ & $(8,2)$ & $\begin{array}{l}\text { (9, } \\
3)\end{array}$ \\
\hline$(9,1)$ & $\begin{array}{c}(10, \\
2)\end{array}$ & $(9,2)$ & $\begin{array}{c}(10, \\
1)\end{array}$ & $(9,2)$ & $\begin{array}{c}\text { (10, } \\
3)\end{array}$ & $(9,3)$ & $\begin{array}{c}(10, \\
2)\end{array}$ \\
\hline$(10,2)$ & $\begin{array}{l}\text { (11, } \\
1)\end{array}$ & $(10,1)$ & $\begin{array}{l}\text { (11, } \\
2)\end{array}$ & $(10,3)$ & $\begin{array}{c}(11, \\
2)\end{array}$ & $(10,2)$ & $\begin{array}{c}\text { (11, } \\
3)\end{array}$ \\
\hline$(11,1)$ & $\begin{array}{c}(12, \\
2)\end{array}$ & $(11,2)$ & $\begin{array}{c}(12, \\
1)\end{array}$ & $(11,2)$ & $\begin{array}{c}\text { (12, } \\
3)\end{array}$ & $(11,3)$ & $\begin{array}{c}(12, \\
2)\end{array}$ \\
\hline$(12,2)$ & $\begin{array}{c}\text { (13, } \\
1)\end{array}$ & $(12,1)$ & $\begin{array}{c}(13, \\
2)\end{array}$ & $(12,3)$ & $\begin{array}{c}(13, \\
2)\end{array}$ & $(12,2)$ & $\begin{array}{c}\text { (13, } \\
3)\end{array}$ \\
\hline$(13,1)$ & $\begin{array}{l}(14, \\
2)\end{array}$ & $(13,2)$ & $\begin{array}{l}\text { (14, } \\
1)\end{array}$ & $(13,2)$ & $\begin{array}{c}\text { (14, } \\
3)\end{array}$ & $(13,3)$ & $\begin{array}{c}\text { (14, } \\
2)\end{array}$ \\
\hline$(14,2)$ & $\begin{array}{c}\text { (15, } \\
1)\end{array}$ & $(14,1)$ & $\begin{array}{c}(15, \\
2)\end{array}$ & $(14,3)$ & $\begin{array}{c}(15, \\
2)\end{array}$ & $(14,2)$ & $\begin{array}{c}\text { (15, } \\
3)\end{array}$ \\
\hline
\end{tabular}

Table 2 : Tensor Product of $P_{15} \times P_{3}$ 


\section{Continuous Monotonic Decomposition of Some Standard Graphs by using an Algorithm}
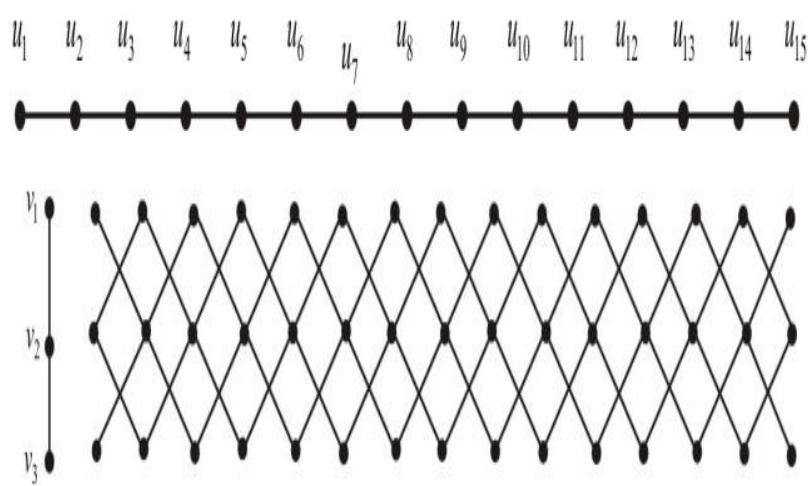

Fig.2.

\section{V.CONCLUSION}

In this paper we described above complete bipartite graphs that accept Continuous monotonic star decomposition. There are many other classes of complete tripartite graphs that accept Continuous monotonic star decomposition. Finally we conclude that in this paper can be extended to complete $m$-partite graphs for grater values of $m$. Also the algorithm can be developed for the tensor product of different classes such as $C_{n}, W_{n}, K_{1, n}$ with $P_{n}$.

\section{CONFLICT OF INTEREST}

The author confirms that there no conflict of interest to declare for this publications.

\section{ACKNOWLEDGEMENT}

The author would like to express his sincere thanks to the Editor-in-Chief for this valuable suggestions towards improvisation of the article.

\section{REFERENCES}

1. Harary, F., Graph Theory. USA: Addison-Wesley Publishing House, 1969

2. Alavi, Y. "The Ascending Subgraph Decomposition Problem" Congress Numerantium, 58 (1987): 7-14.

3. Gnanadhas, N., and Paulraj Joseph, J. "Continuous Monotonic Decomposition of Graphs." International Journal of Management and Systems, 16 (2000): 333-344.

4. Gnanadhas, N., and Paulraj Joseph, J., "Continuous Monotonic Decomposition of Cycles." International Journal of Management and Systems, 19 (2003): 65-76.

5. Nagarajan, A., and Navaneetha Krishnan, S., "Continuous Monotonic Decomposition of some special class of Graphs." International Journal of Management and Systems, 21(2005): 91-106.

6. Joseph Varghese, and AntonysamyMapana, A., Journal of Sciences, 8(2) (2009): 7-19.

7. Sudarsanam, N., and Sreedharan, N., "On Edge decomposition of graph." National Workshop on Graph Theory and its Application, Feb.21-27, (1996): 203-206.

8. Asha, S., and Kala, R., "Continuous Monotonic Decomposition of some special class of graph." Int. Journal of Math. Analysis, 4(51) (2010): 2535-2546.

\section{AUTHORS PROFILE}

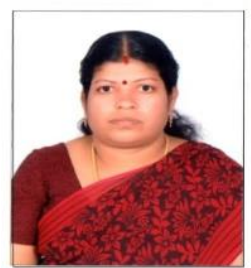

Dr. C. Sujatha a well-known academician has taken the charge as principal of Marudupandiyar College, Thanjavur, Since December 2018. She had obtained her doctoral degree from Bharathidasan University Tiruchirappalli, which is ranked among the top world universities. She is Mathematician by profession and specialized in Graph theory and applications. She has more than

15 years of teaching and 7 years of research experience. She has published many research articles in reputed in International Journals and conferences and also organized many Conference,

Published By:

Seminar and Workshops. She has the vision of fostering academic learning and research among students and research scholars for the development of innovative concepts and products to the betterment of society. She firmly believes that quality education can only lead to innovations and new product developments. She her wide and rich administrative research and international exposure to achieve the vision and Build Institutions.

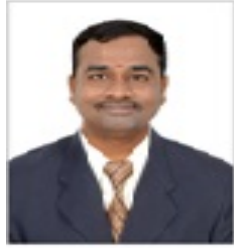

Dr. A. Manickam, Associate Professor in the Department of Mathematics has 10 years of teaching and research experience, including 8 years of research experience in the field if Mathematical Modeling and Graph theory and Applications. He has received both his B.Sc., Degree in Mathematics, M.Sc., Degree from Bharathidasan University, B.Ed., Degree from Tamil Nadu Teachers Education University and M.Phil., Ph.D from the Bharathidasan University.He has published over 35 research publications in referred International Journals and in proceedings of international conferences. $\mathrm{He}$ is a recognized supervisor of Bharathidasan University. His current research work includes, Mathematical modeling deal with Differential Equations. He has rganized 05 International onference, seminar and workshops. He Has acting as a reviewer for 06 International peer reviewed Journals. (Synergistically activities).

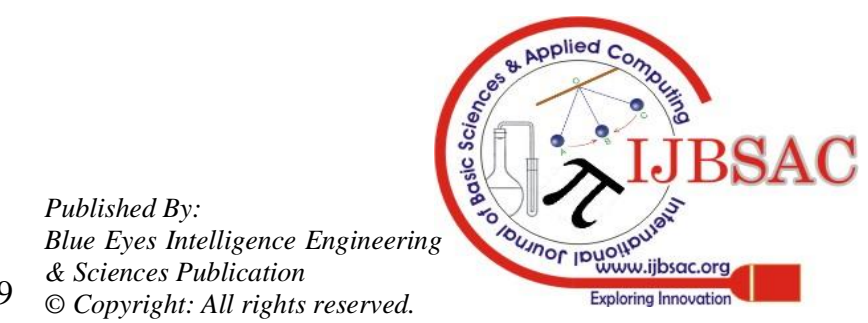

